\title{
"Partigiani..." Storie di resistenza nel Parmense: gli studenti raccontano
}

L'Istituto storico della resistenza di Parma e sei amministrazioni comunali della provincia hanno promosso un percorso storico-didattico per giovani studenti con l'obiettivo di indagare l'identità di coloro che settant'anni fa aderirono alla Resistenza contro i nazifascisti, i luoghi in cui operarono, gli eventi che li videro protagonisti. La ricerca, che ha messo gli alunni a diretto contatto con le fonti storiche, ha condotto alla realizzazione di quaderni didattici.

The Istituto storico della Resistenza and six municipal councils in the province of Parma promoted a didactic project for young students, which aim was to investigate the identity of those who seventy years ago joined the Italian Resistance movement and fought against the Nazi-fascists, the areas where they operated, the events they took part to. Scholars were put in direct contact with the historical sources and eventually produced didactic books about the topic.

(Contributi video disponibili online: 目国 https://www.youtube.com/ watch? $\mathrm{v}=4 \mathrm{XYc}-\mathrm{pSZzVo}$ ).

\section{Introduzione}

A 70 anni dalla liberazione dell'Italia dall'oppressione nazi-fascista, l'Istituto storico della resistenza e dell'età contemporanea di Parma si è fatto promotore di un percorso storico-didattico che avesse come obiettivo l'indagine dell'identità di coloro che aderirono alla lotta di Liberazione, i luoghi in cui operarono e gli eventi che li videro protagonisti. Al progetto, riservato alle scuole secondarie di 
primo grado della provincia di Parma, hanno aderito le classi terze dei comuni di Collecchio, Fontanellato, Lesignano de' Bagni, Sala Baganza, San Secondo e Torrile.

Attraverso l'utilizzo di testimonianze, documenti d'archivio, immagini e carte topografiche gli studenti sono stati chiamati a ricostruire l'esperienza di un determinato gruppo di partigiani provenienti da un preciso comune o frazione seguendone gli spostamenti, individuandone le aree occupate, ricostruendo i combattimenti e i rastrellamenti nei quali furono coinvolti. Queste attività, svolte durante tutto l'anno scolastico, sono sfociate nella realizzazione di quaderni didattici che hanno raccolto il lavoro realizzato dagli studenti e che sono stati presentati nei rispettivi comuni durante le celebrazioni del 25 aprile 2015.

\title{
2. Obiettivi del progetto
}

\author{
Nessuna conquista è per sempre: \\ c'è sempre qualcuno che è interessato \\ a toglierla, per cui resistere è non solo un dovere, \\ ma anche una necessità dei giovani d'oggi, \\ altrimenti non si va vanti. \\ (Maria Cervi, figlia di Antenore).
}

Le amministrazioni comunali, in collaborazione con le sezioni Anpi locali, hanno raccolto l'invito a promuovere e sostenere attività e progetti che aiutassero $i$ cittadini più giovani a riflettere e a prendere coscienza del percorso, faticoso $\mathrm{e}$ doloroso, che ha portato alla nascita della Repubblica italiana e che ha visto strettamente collegati valori come Resistenza e impegno di cittadinanza: «la scelta di presentare ai più giovani la Resistenza, attraverso la vita dei protagonisti e degli eventi che sono accaduti nei luoghi dove loro stessi stanno crescendo, è senz'altro una sfida pregnante, urgente e difficile». Questi sono stati gli obiettivi, nelle parole dei referenti del progetto per il comune di Collecchio; mentre per Lia Musumeci, assessore servizi ai socio-educativi del comune Lesignano, la «crisi della politica, la disgregazione delle ideologie, la crisi degli ideali, rendono ancora più necessario trovare una fonte condivisa di valori; i giovani altrimenti rischiano di crescere senza riferimenti e il risultato non può che essere una democrazia debole, precaria, formale». Le insegnanti delle scuole coinvolte, a loro volta, hanno voluto riprendere in mano il valore "educante" della storia stimolando i propri alunni alla curiosità, all'ascolto di testimonianze, alla ricerca documentaria e alla rielaborazione dei pensieri che da tali attività scaturivano. 
L'Isrec di Parma ha proposto un vero e proprio laboratorio didattico: la prima parte del percorso è stata dedicata ad un inquadramento storico generale, che aiutasse i ragazzi a familiarizzare con il contesto da cui era scaturito il movimento resistenziale a partire dall' 8 settembre 1943. Successivamente la lente è stata spostata su Parma e sulla sua provincia: partendo dagli Appennini e scendendo fino alla pianura a ridosso del fiume Po, le ricerche si sono concentrate sulle storie delle formazioni partigiane di tutto il territorio parmense.

\section{Le questioni}

Le prime domande a cui hanno tentato di dare risposta le ricerche dei giovani studenti hanno interessato le biografie degli uomini e delle donne della Resistenza: chi erano? In quanti parteciparono? Da quale classe sociale provenivano? Quale la loro età e quindi anche la loro esperienza? Grazie all'analisi di ruolini e dei diari storici delle brigate sono emersi dati che hanno tracciato le linee di un quadro complesso, diversificato a seconda delle zone ma soprattutto nuovo agli occhi di questi giovani "ricercatori".

Si legge, ad esempio, tra le pagine redatte dai ragazzi di Lesignano:

Analizzando le date di nascita dei partigiani abbiamo rilevato che avevano un'età media tra i 23 e i 24 anni [...] Dall'elenco fornitoci abbiamo notato che il più vecchio era nato nel 1900 mentre la più giovane partigiana era una donna, Riccò Gina, nata nel 1930 e caduta in combattimento nel 1945. La sua sorte ci ha molto colpito. Insieme a lei si unirono altre sei donne. Abbiamo poi cercato altre notizie in testi che parlavano di cosa significasse essere uomini e donne durante il fascismo e perché molti decisero di unirsi alla Resistenza. Di una delle testimonianze raccolte ci è sembrata significativa questa frase: "Quando mi faceva gridare «viva il duce» non lo volevo fare e lei [la maestra] mi picchiava. Anche mio fratello ha preso delle botte, quindi fin da piccolo noi non li sopportavamo".

Gli alunni delle scuole di Fontanellato, rileggendo le testimonianze di alcuni partigiani, hanno potuto rilevare che il primo nucleo di antifascisti del paese «era composto da uomini di diverse idee politiche, comunisti e cattolici. Si andava da monsignor Grassi per accordarsi su come costruire un gruppo ben organizzato, che si costituirà poi nell'aprile 1944».

A destare particolare curiosità è stato anche il tema delle donne partigiane. Così hanno scritto i ragazzi delle scuole medie di San Secondo:

[...] abbiamo notato che il numero delle donne era davvero esiguo ma ciononostante esse seppero dare un contributo importante alla Resistenza. Alcuni uomini furono coinvolti nella lotta clandestina proprio dalle donne: è il caso del partigiano Felice Costa che partecipò alla sua prima azione incoraggiato da un'amica di fami- 
glia, Adele Zucchi. Di Adele, nome di battaglia "Anna", ci ha colpito che aderì alla Resistenza insieme ai fratelli Felice ed Oscar, tutti figli di Mario Zucchi: un'intera famiglia partigiana! [...] nell'inverno tra il ' 44 e il ' 45 le donne sentirono sempre più impellente la necessità di aiutare i partigiani così; sebbene poche di loro impugnarono le armi, diedero una mano in altro modo. Ricordiamo qui un aneddoto: per la festa dell'Epifania fascista, durante la quale era usanza raccogliere abiti e giocattoli da donare alle famiglie più bisognose, alcune donne di San Secondo andarono di casa in casa per raccogliere sì vestiti e biancheria che vennero però destinati ai partigiani rifugiatisi in montagna.

L'analisi storica si è poi soffermata sulle storie dei partigiani caduti, i cui nomi sono ricordati nelle vie e nei diversi monumenti dei loro paesi. Da qui sono scaturite le riflessioni di alcuni ragazzi di Lesignano:

Nei libri di storia non vengono mai ricordate tutte le persone che hanno dato la vita per la libertà. Molti sono morti in battaglia, altri durante i rastrellamenti nazifascisti. Nel nostro piccolo vorremmo ricordare quelli del nostro paese $[\ldots]$ quelli che sono stati incarcerati, torturati e poi fucilati. [...] Vorremmo nominare anche i russi che hanno aiutato i nostri partigiani a Lesignano e che sono morti durante la battaglia del settembre $1944[\ldots]$.

Sulla stessa linea si sono posti anche gli studenti di San Secondo:

Tra i caduti vanno ricordati anche quei civili che a maggior ragione furono vittime ingiuste di una guerra senza senso. Tra questi ci furono anche dei bambini: nell'aprile del '45 un aereo americano che sorvolava le campagne della Bassa per colpire i tedeschi in ritirata, sganciò una bomba non lontano da una bambina che per evitala si gettò in un fosso dove venne ricoperta dalla terra smossa dalla deflagrazione e ne morì soffocata.

L'attenta lettura di alcuni documenti ha permesso poi ai "giovani storici" di inserire i volti, le singole storie dei partigiani, le loro azioni di lotta in un contesto di appartenenza: l'analisi delle brigate, dei distaccamenti, delle zone in cui hanno operato ha consentito di ricostruire una mappa attendibile della Resistenza nel territorio parmense.

Tali questioni, inerenti perlopiù alle biografie degli uomini e delle donne che aderirono alla lotta clandestina, non hanno esaurito le ricerche dei ragazzi i quali, attraverso un'analisi approfondita delle fonti, hanno anche sondato le relazioni tra i partigiani e la popolazione civile, riconoscendo a quest'ultima un'imprescindibile ed intensa attività di supporto alla lotta di liberazione nazionale.

\section{I documenti e le fonti}

Le ricerche si sono avvalse di fonti diversificate. I ricercatori dell'Isrec hanno 
messo a disposizione alcuni importanti documenti d'archivio: ad esempio sono state fondamentali (sia per il laboratorio di Fontanellato che per quello di San Secondo) le pagine del Diario storico della 78sima Brigata SAP scritte dal comandante Annibale Ballarini, nome di battaglia "Bongiorno", che hanno permesso agli alunni di trovare agevolmente risposta alla maggior parte delle loro questioni iniziali. Di grande rilievo storico, e molto interessanti per gli occhi attenti dei ragazzi, i ruolini di brigata: tali elenchi sono stati la base di analisi anche "statistiche", ad esempio sull'adesione femminile alla lotta, sull'età media dei partigiani, sulla partecipazione, per gli uomini, alla leva e/o al precedente conflitto mondiale, sui nomi dei caduti, ecc. In alcuni casi anche le rare ma pur sempre preziose testimonianze orali di partigiani ancora in vita hanno permesso una relazione di maggiore prossimità tra i giovani ricercatori di oggi e i giovani partigiani di ieri.

\section{Riflessioni sulla Resistenza}

Obiettivo cardine di ogni percorso storico-didattico dell'Isrec Parma - e quindi anche di questo - è innescare riflessioni personali ed autentiche e stimolare la coscienza attraverso la conoscenza della storia. Le pubblicazioni finali hanno raccolto i pensieri dei partecipanti: ognuno, con le parole (a volte anche poetiche) e con le immagini, ha potuto esprimersi in assoluta libertà. Ai fini di un'onesta restituzione del progetto "Partigiani..." ci è sembrato importante riportarne alcuni per rendere merito al lavoro dei ragazzi.

«La cosa che più mi ha colpito di questa ricerca è che non pensavo che un paese così piccolo come Lesignano potesse avere una storia così grande» (Luca - Lesignano).

«La libertà è stato il primo motore che ha azionato la forza partigiana» (Martina e Lucrezia - San Secondo).

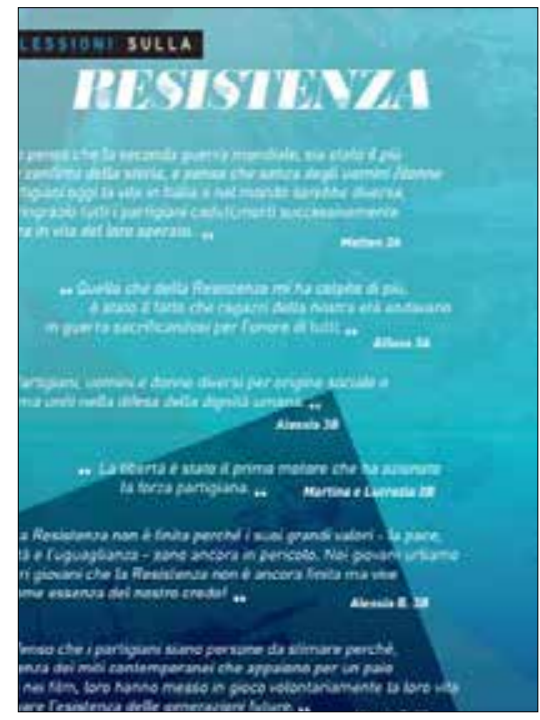

Una pagina di riflessioni degli studenti.

«La Resistenza non è finita perché i suoi grandi valori - la pace, la libertà e l'uguaglianza - sono ancora in pericolo. Noi giovani urliamo ad altri giovani che la Resistenza non è ancora finita ma vive in noi come essenza del nostro credo!» 
(Alessia - San Secondo).

«Fra i partigiani si sono distinte le donne, combattenti all'ombra, ma unite nel nome della libertà, con costanza e dedizione fino al termine dei loro giorni» (Alessandro - San Secondo).

\section{Poesia}

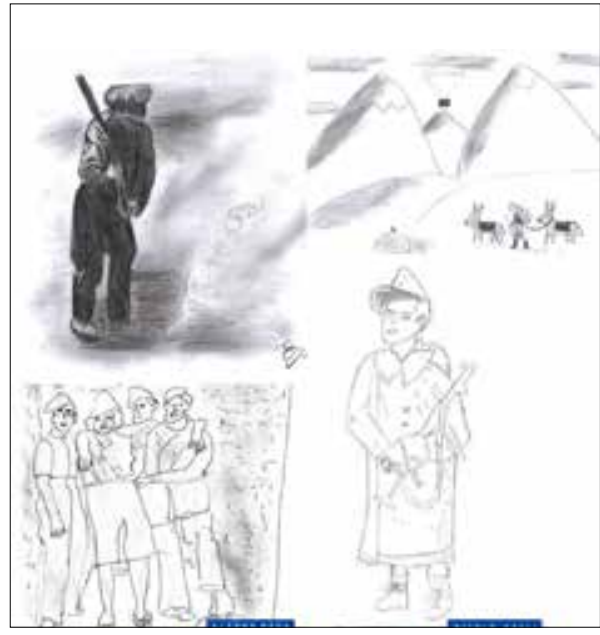

La Resistenza disegnata dagli studenti.

Resistere a Fontanellato

(di Alessia, Alessandro, Tisha, Sukhmander, Mattia - Fontanellato)

I defunti furono tanti

le condizioni erano umilianti, troppo sangue è stato sparso in questo campo riarso.

Athos, Fulmine e Pantera

i nomi di battaglia di una vecchia era

tante eran le brigate

ove anche le donne alla morte condannate.

Ebbe inizio a Fontanellato la Resistenza

che massacrò di ogni persona l'esistenza

nella $78^{\circ} \mathrm{SAP}$ erano tanti giovani

tornarono dalla guerra e diventarono uomini.

Combatterono per la propria patria con gloria ecco perché noi dobbiamo averne memoria costruiamo l'amore e non la guerra si sta meglio per mano che sotto terra. 